\title{
STABILITY OF EMISSION-LINE CLOUDS IN SEYFERT GALAXIES
}

\author{
William G. Mathews and Sylvain Veilleux \\ Lick Observatory \\ University of California \\ Santa Cruz, CA 95064 \\ USA
}

\begin{abstract}
Otherwise acceptable kinematic and dynamic models for emission line regions may be ruled out if the line-emitting clouds are unstable. We discuss the stability of radially-moving clouds to lateral gas flows. Such flows result from internal overpressures arising from the absorption of continuum radiation.
\end{abstract}

Emission line clouds in quasars and active galaxies can be disrupted by a variety of instabilities. While disruption of clouds may not eliminate line emission from fully-ionized clouds, emission lines produced in optically thick regions (Mg II, Fe II, O I, etc.) will not be present if the clouds are unstable. Since emission features from optically thick zones are common to both broad and narrow line regions, the requirement of cloud stability sharply constrains otherwise acceptable kinematic or dynamic models of emission line regions.

Here we emphasize an instability that occurs in all radially-moving emission line clouds. When continuum radiation is absorbed preferentially near the irradiated surface, a small compressive force acts on the cloud in the radial direction. This compression causes a radial pressure maximum in the cloud which cannot be balanced by pressure variations in the local cloud-confining medium. The gas in the cloud must therefore flow out and expand transverse to the radiation flux vector. A similar cloud disruption by lateral flow can occur if the cloud is "derimmed" as discussed by Mathews ( $A p . J ., 305,187$ ), but only overpressure-driven lateral flows are considered here. We consider only radially-moving clouds: non self-gravitating, orbiting clouds are subject to a variety of additional instabilities such as Keplerian shear and differential Poynting-Robinson drag and are unlikely to remain intact for a single orbit in many cases.

The relatively short disruption time for the overpressure-driven lateral flow instability implies that clouds must be rapidly accelerated through emission line regions before they erode away: Large clouds are obviously adrantageous.

Although the overpressure-driven lateral flow instability must occur in both broad and narrow line clouds. we consider here as an example the stability requirements of narrow line emission clouds for which the physics and kinematics are better understood. We are also interested in cases in which the clouds experience a dynamic pressure on their irradiated surface: this could apply to clouds that are accelerated 
radially outward by a wind or to clouds infalling upstream into a wind. In the absence of radiative momentum effects, such clouds are always Rayleigh-Taylor unstable on timescales short compared to their lifetime in the line-emitting region. Paradoxically, the internal radial pressure maximum arising from the absorption of radiation renders clouds accelerated by dynamic pressure Rayleigh-Taylor stable although some lateral flow must also occur. Rayleigh-Taylor stability occurs since the sense of the local effective gravity at the pushed surface is reversed by the absorption of radiation and is directed from the (less dense) intercloud wind toward the (denser) clouds.

For small dynamic pressures on the irradiated surface, a typical cloud is unstable to lateral flow on times short compared to the radial flow time in the line-emitting region. As the dynamic pressure increases, however, the cloud becomes effectively stable to lateral flow while moving through the line-emitting region since the radial extent of the overpressure zone in the cloud is reduced along with the lateral flow rate. If a critical value of the dynamic pressure is exceeded, however, the clouds no longer have an overpressure but are disrupted instead by the Rayleigh-Taylor instability.

We adopt the following empirically determined cloud properties for the narrow line region: cloud density $n=10^{6} \xi^{-p} \mathrm{~cm}^{-3}$ (with $p=0.5$ or 1.5 ), cloud velocity $|u|=500 \xi^{-0.1} \mathrm{~km} \mathrm{~s}^{-1}$, and incident spectrum $F_{\nu} \propto \nu^{-1.4}$ where $\xi=R / 3 \mathrm{pc}$.

For these typical narrow line parameters, only clouds having column densities $N \gtrsim 10^{23} \mathrm{~cm}^{-2}$ can survive the lateral flow instability as they move through the emission line profile in the absence of dynamical pressure. (This is close to the largest possible narrow line clouds; those having $N \gtrsim 10^{24}$ are too large to fit in the narrow line region.) However, for a stronger dynamical pressure (half that required for the clouds to become Rayleigh-Taylor unstable), clouds of smaller columns $N \approx 10^{22}$ can be made effectively stable at $R \approx 3 \mathrm{pc}$. The transition to Rayleigh-Taylor instability does not occur until the dynamic pressure is quite large, typically $10-100$ times the local isotropic pressure in the cloud-confining medium.

These conclusions apply to both radially inflowing or outflowing clouds. For the case of outflowing clouds, optically thick [O I]-emitting clouds can be coherently accelerated by a wind without disruption. Clouds having $N \gtrsim 10^{21} \mathrm{~cm}^{-2}$ could also be optically thick to dust absorption. In this case the blueward asymmetry observed in [O I] could arise from a source of (dust) occultation near the center of the cloud system provided the [O I] emission is radiated approximately isotropically from individual clouds. In a similar manner, optically thick clouds falling into a wind or stationary atmosphere can be stabilized to Rayleigh-Taylor disruption by the absorption of radiative momentum at the inner surface.

Since most radially moving clouds are either Rayleigh-Taylor unstable or unstable to lateral flows, a significant number of small optically thin clouds must always result from the debris of larger, more stable clouds that are known to be present. The contribution of these debris fragments to the emission line profiles is unclear. 